\title{
Considerations for virtual surgical planning and simulation in orthognathic surgery-a narrative review
}

\author{
Tae-Geon Kwon, Sung-Tak Lee, So-Young Choi, Jin-Wook Kim \\ Department of Oral and Maxillofacial Surgery, School of Dentistry, Kyungpook National University, Daegu, Republic of Korea \\ Contributions: (I) Conception and design: TG Kwon; (II) Administrative support: SY Choi, JW Kim; (III) Provision of study materials or patients: TG \\ Kwon, SY Choi, JW Kim; (IV) Collection and assembly of data: TG Kwon, ST Lee, JW Kim; (V) Data analysis and interpretation: TG Kwon, ST \\ Lee, SY Choi; (VI) Manuscript writing: All authors; (VII) Final approval of manuscript: All authors. \\ Correspondence to: Tae-Geon Kwon, DDS, PhD. Department of Oral and Maxillofacial Surgery, School of Dentistry, Kyungpook National University, \\ 2177 Dalgubeol-daero, Jung-gu, Daegu 41940, Republic of Korea. Email: kwondk@knu.ac.kr.
}

Background and Objective: Although there are many benefits to use 3D virtual surgical planning (VSP)
for orthognathic surgery, there are only a few reports on the potential errors or mistakes that can occur with
VSP and simulation processes. This narrative review updates information on VSP and discusses the various
precautions and considerations for VSP in orthognathic surgery.
Methods: In these reviews, PubMed data base search was performed following terms: Orthognathic
Surgery, Computer-assisted, computer-aided, virtual planning, CAD-CAM. The published reports from Jan
2000 to April 2020 in English were included. Study with full-text were selected and reviewed.
Key Content and Findings: Application of VSP allows visualization of the interosseous relationship,
segment overlap or bony interference, and significant interosseous gaps. Potential sites for additional bone
grafts or bone reduction can be anticipated. Additionally, presurgical planning can be performed with
unlimited time. Since most VSP is performed by an outsourced company, quality assurance in VSP depends
on a third-party, and sometimes there is lack of clarity when delineating the responsibilities for surgical
outcomes. To increase the precision of 3D planning and simulation, surgeons need to be aware that the
VSP cannot automatically ensure the success of surgical outcomes. Errors can occur at every step, such as
integration of $3 \mathrm{D}$ dentition to skull data, segment identification and mobilization, computer-aided surgical
simulation, fabrication of splint and surgical guides, 3D image superimposition, and determination of
occlusion in virtual space.

Conclusions: Thorough understanding of the source of errors in VSP can lead to successful surgical outcomes in orthognathic surgery performed with VSP.

Keywords: virtual surgical planning (VSP); simulation; orthognathic surgery; three-dimensional (3D)

Received: 20 August 2020; Accepted: 11 September 2020; Published: 10 November 2020.

doi: $10.21037 /$ fomm-20-54

View this article at: http://dx.doi.org/10.21037/fomm-20-54

\section{Introduction}

In patients with facial deformities, such as malocclusion, surgical planning and simulation of surgical outcomes are the most important processes in presurgical workups for successful orthognathic treatment. It is necessary to correctly move the osteotomized segment and dentition to the required position in $3 \mathrm{D}$ space according to the preoperatively determined surgical plan. Therefore, intraoperative control of precise and accurate mobilization of osseous segments is emphasized. Currently, computerassisted virtual surgical planning (VSP) has been widely introduced in various institutions and it is commonly used in oral and maxillofacial surgery, especially in the field of orthognathic surgery (1-4). Three-dimensional (3D) VSP is widely accepted as mainstream for treatment 
planning compared to the previous classical 2D planning or articulator model surgery $(4,5)$. The accuracy and feasibility of VSP for orthognathic surgery is well documented in the literature (6-9). Using computerized treatment planning, it is possible to perform virtual segmentation of the maxilla or mandible and repositioning of the osteotomized segment is therefore possible. This enables precise mobilization of the horizontal, vertical, and transverse direction segments with six degrees of freedom according to the planned movements. At the same time, it is possible to visualize the interosseous relationship, segment overlap or bony interference, and significant interosseous gaps and potential sites for additional bone grafts or bone reduction. Another advantage is that the trial of presurgical planning can be performed within an unlimited time.

Technological advancements enable not only virtual planning but also intraoperative utilization of surgical splints or surgical guides fabricated by a 3D computeraided design/computer-aided manufacturing (CAD/ CAM) system. A step-by-step protocol for 3D VSP is well established at each institute even though there are some minor technological differences. Overall, the protocol is composed of several steps: (I) acquisition of computer tomography (CT) or cone-beam computed tomography (CBCT) images of maxillofacial structures and scanned maxillomandibular dental casts; (II) 3D image segmentation, fusion, and 3D superimposition of dental arches in CT images for virtual planning; (III) implementation of virtual treatment planning based on the diagnosis and surgeon's plan for the surgery; (IV) fabrication of CAD/CAM-utilized surgical splints or surgical guides; (V) intraoperative utilization of surgical splints or surgical guides-surgical transfer of planned surgical movements; and (VI) postoperative validation of the VSP compared to real postoperative results $(1,10,11)$. Recently, surgical guides accompanied by pre-bent titanium plates (12) or patient-specific, 3D printed plates are being used with (13) or without an intermediate splint (14).

Since the osteotomized segments of the maxilla can be mobilized to complex degrees and directions, 3D planning and simulation are very helpful for accurate $3 \mathrm{D}$ simulation. Previous reports have shown that the application of VSP demonstrated acceptable surgical accuracy in one-piece maxillary osteotomy (15-18) or multipiece maxillary orthognathic surgeries (19-24). Many previous publications have emphasized and highlighted the accuracy of a 3D planning system using the various software programs and hardware. However, the enthusiasm for 3D technology sometimes underestimates or ignores the potential source of errors during virtual planning. Moreover, a systematic review of virtual planning in orthognathic surgery suggested that even though there has been no reported financial conflict of interest, there is a risk of financial bias in some studies because of the possibility of financial gains from 3D software development and application (7).

In this narrative review, the studies suggesting quantitative data is included to reduce the bias of the publication. Since the VSP is recently shown in oral and maxillofacial areas, publications showing important concepts or explaining operating process of VSP were also reviewed.

Although there are many benefits to using 3D VSP for orthognathic surgery, there are only a few reports on potential errors or mistakes that can be encountered with VSP and simulation processes. The key questions in this review was as follows: (I) which process during VSP can be the source of errors? (II) what is different between in-house and outsourced VSP? To answer these two key questions, this review aims to update information on VSP and discuss the various precautions and considerations in VSP for orthognathic surgery.

We present the following article in accordance with the Narrative Review Checklist (Available at https://fomm. amegroups.com/article/view/10.21037/fomm-20-54/rc.

\section{Methods}

In these reviews, PubMed data base search was performed following terms: Orthognathic Surgery, Computer-assisted, computer-aided, virtual planning, CAD-CAM. The published reports from Jan 2000 to April 2020 in English were included. Study with full-text were selected and reviewed.

\section{Discussion}

\section{Practical advantage of computerized surgical planning in orthognathic surgery}

VSP can facilitate the quantitative analysis of outcomes and provide greater accuracy in orthognathic surgery (6). Therefore, computerized planning and simulation for orthognathic surgery would allow surgeons to perform more accurate and efficient orthognathic surgeries $(8,25)$. There are various advantages to VSP-modulated orthognathic surgery.

First, more accurate and quantitative analysis of 

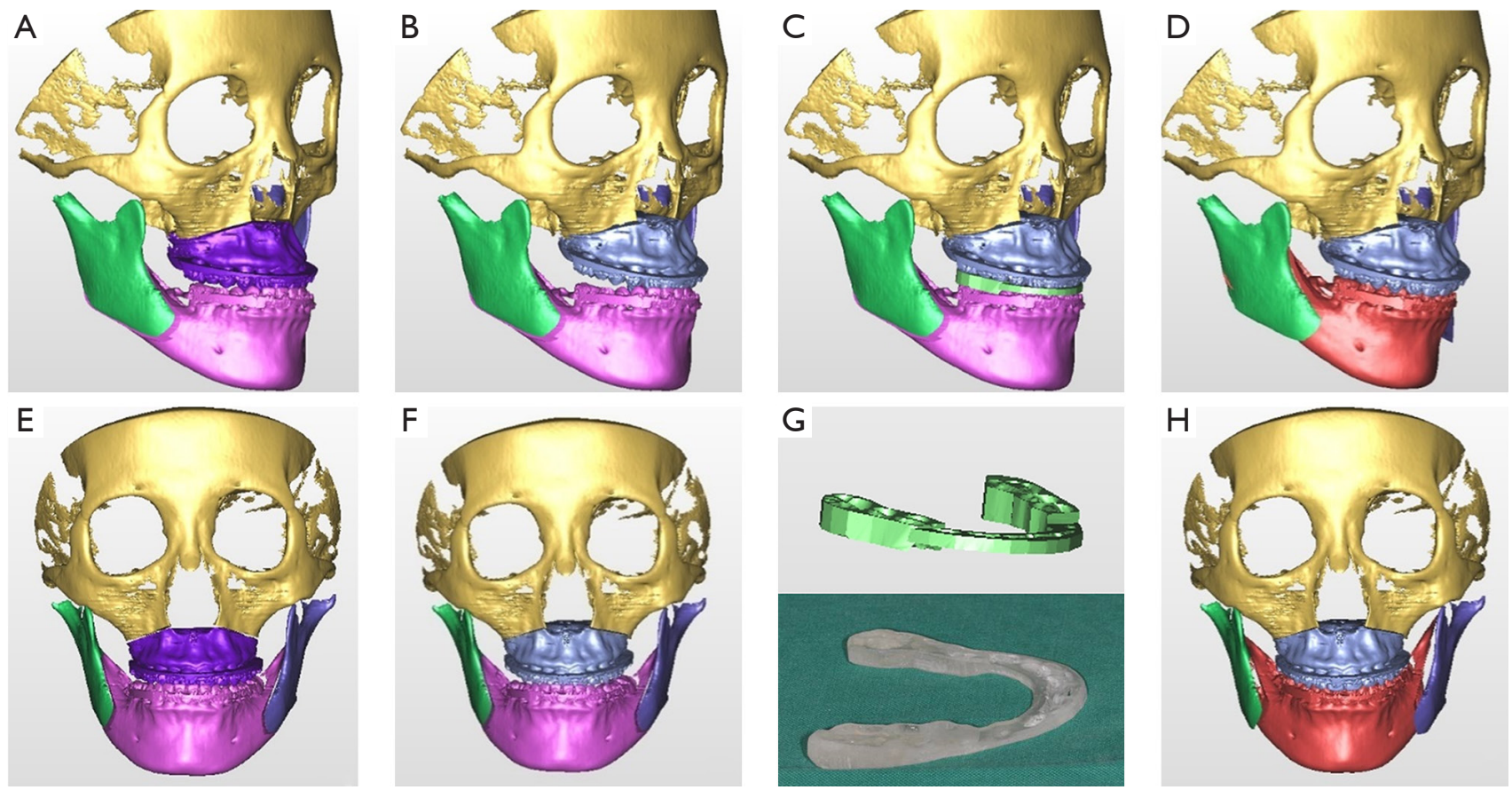

Figure 1 Fabrication of a computer-aided design/computer-aided manufacturing (CAD/CAM) intermediate splint for two jaw surgery. (A) Laser scanned dental cast data and cone-beam CT data were superimposed in virtual space. (B) Maxillary repositioning was performed according to the surgical plan. (C) virtual fabrication of an intermediate splint and 3D printed splint. (D) Simulated position of mobilized maxillomandibular structures.

the various deformities is possible with VSP, which previously were impossible with $2 \mathrm{D}$ cephalometric analysis. Computerized planning in orthognathic surgery allows surgeons to carry out comprehensive evaluation of anatomical structures of the surgical field. In asymmetric patients, VSP can be used to diagnose presurgical problems and can predict the postoperative $3 \mathrm{D}$ position of osteotomized segments, thereby predicting redundant asymmetry after surgery (26). At the same time, the need for dental decompensation after surgery can also be visualized and it is possible to reflect the magnitude of overcorrection to VSP in advance (1).

Another advantage is that VSP can allow minimally invasive surgery. VSP follows the osteotomy line nearly the same as in a real intraoperative osteotomy. Repetitive virtual surgery for the best possible results in problematic cases can minimize the surgical errors and can allow the surgeon to accurately reproduce the surgical plan. At the same time, surgeons can anticipate potential intraoperative problems and reduce complications using VSP.

Additionally, residents can participate in the planning procedure and can have deeper insights on the surgery being planned. Patients can have sufficient information on the surgery and can understand postoperative sequelae more easily. It is very useful for patient and student education.

Validation of the surgical outcome can be carried out with postoperative assessment. Since all the data related to computerized surgical planning and postoperative outcomes are stored in a database, these accumulated quantitative follow-up data would be helpful for developing an improved protocol or technical innovations for further treatments.

\section{Potential source of errors in computer-assisted planning and surgical applications}

Since the actual surgical result is significantly influenced by the simulated maxillary position in virtual surgery or articulator model surgery, the intermediate splint or surgical guide is important and this core step can determine the accuracy of the surgery (Figure 1). The many steps of a conventional articulator-based model surgery allow a high probability of errors. The step of taking an impression, bite registration, facebow transfer, maxillary repositioning and 


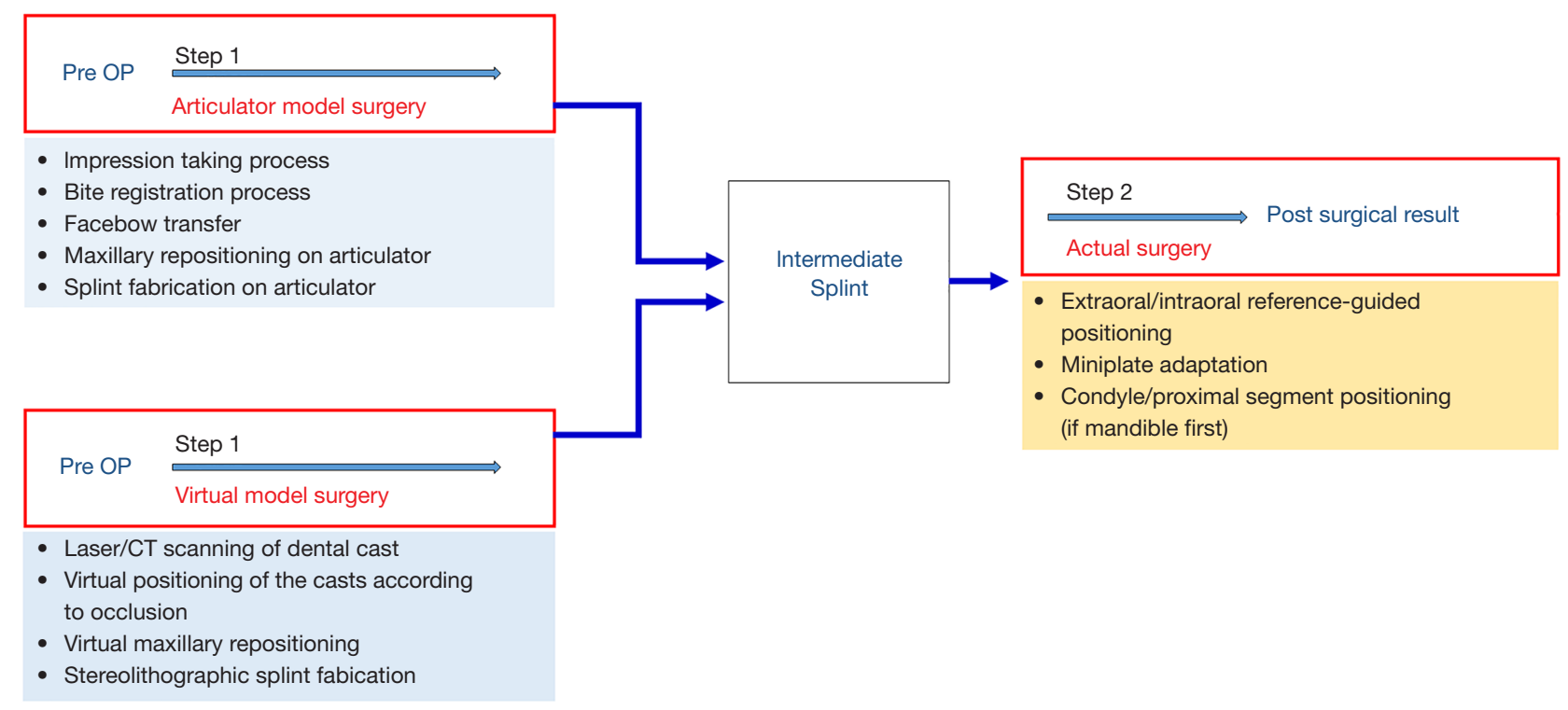

Figure 2 Comparison of potential errors related to classical articulator model surgery $v s$. virtual model surgery.

splint fabrication on an articulator can be sources of errors (Figure 2). However, a VSP does not directly guarantee surgical accuracy. Inaccuracy associated with a computerized planning procedure exists for many factors; e.g., head positioning, 3D data integration, segment positioning, and the stereolithographic splint fabrication procedure.

\section{Errors in 3D data integration and fusion}

It is important to integrate the $3 \mathrm{D}$ data of dentition and the skeleton from different imaging techniques to create a $3 \mathrm{D}$ virtual dentofacial skeleton in the same virtual space. Because orthodontic brackets, metallic restorations, and a prosthesis are usually installed in many patients, metallic artifacts in CBCT images are a significant problem (27). Therefore, dentition is usually determined with laser surface scanning and it requires image fusion from skeletal CT scans. Many technical developments have been reported to overcome radiographic artifacts that can interfere with the optimal integration of maxillomandibular dentition using CT images. Multiple scans $(28,29)$, fiducial markers (19,30-32), registration blocks $(19,33)$, surface matching (34-37), voxel based registration (29), or a specialized algorithm using a combination of various methods (38) are suggested to improve the accuracy of $3 \mathrm{D}$ data integration of dentition and the skeleton. Currently, the function of point or surface registration in $3 \mathrm{D}$ planning software is used often (39). The dental arch information STL file format is usually introduced in $3 \mathrm{D}$ simulation software.

The registration process is performed by: (I) matching corresponding reference points that are close to each other, (II) using regional surface-based registration (3D \& MPR images), and 3) validating the superimposition accuracy (Figure 3). However, other factors remain a concern; e.g., (I) the time interval between obtaining the dental impression or intraoral scan vs. CBCT imaging because of potential effects of presurgical orthodontic movements; and (II) the intermaxillary occlusal relationship between the dental cast vs. CBCT (centric relation or centric occlusion bite). Complete, perfect integration of an individual maxillary or mandibular dental cast to CBCT is not always possible but it has clinically acceptable accuracy with current technology.

\section{Errors in the virtual planning process-head positioning, segment positioning}

The head position in virtual space is important for treatment planning. The vertical position reference landmarks or occlusal plane are especially important for maxillary mobilization (Figure 4). Moreover, the 3D reference plane in virtual surgical movements should coincide with the real pre-postoperative head position. Otherwise, validation of the $3 \mathrm{D}$ VSP is not possible. The $3 \mathrm{D}$ reference plane is frequently defined by anatomical landmarks, such as Frankfort's horizontal plan (40). The natural head position can also be used in VSP but has some 


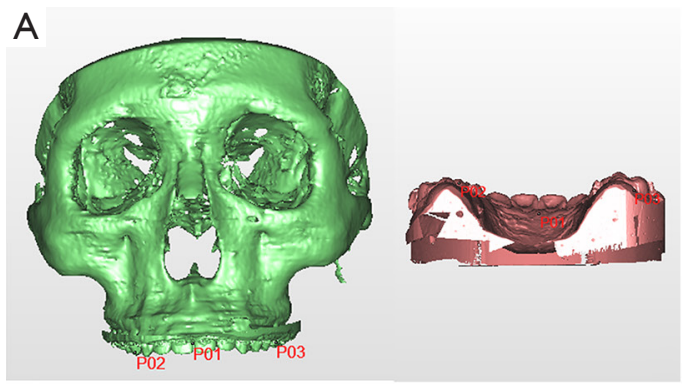

C

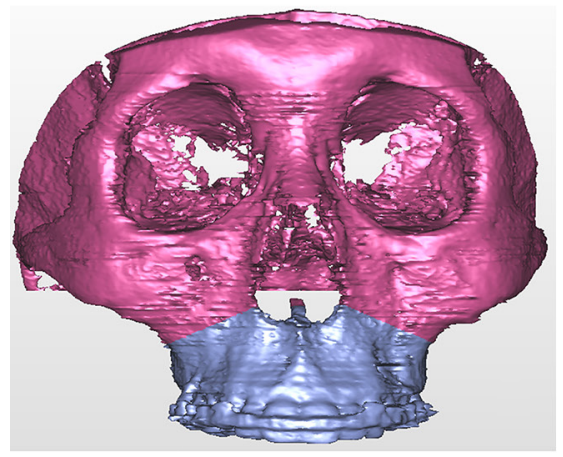

D

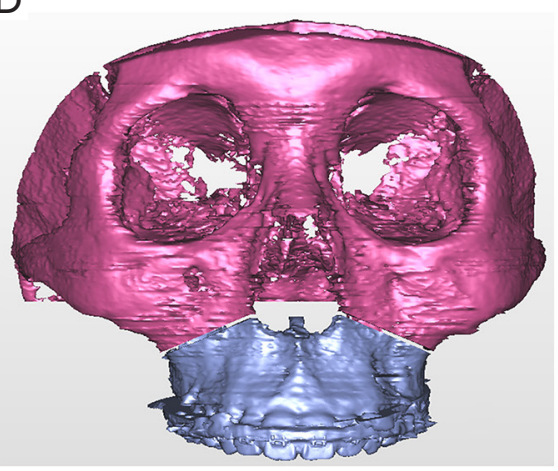

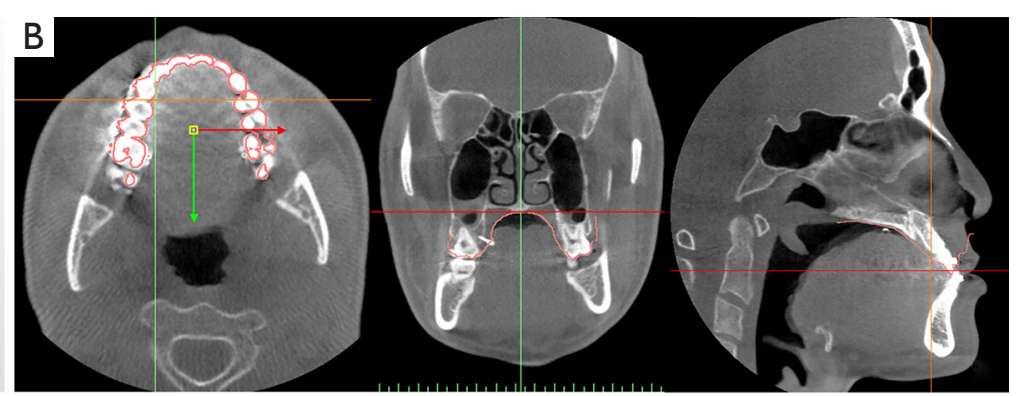

E

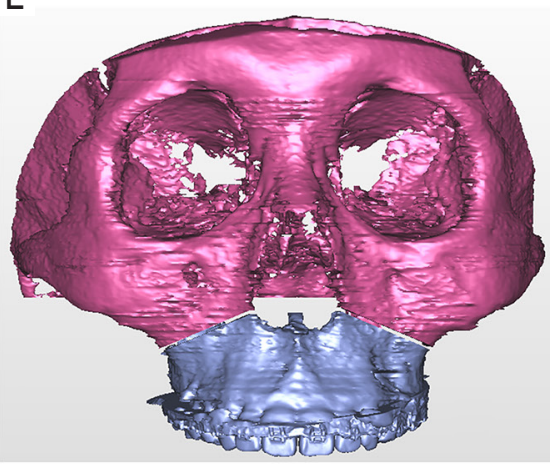

Figure 3 Step-by-step procedure for image fusion of dental model and 3D cone-beam computed tomography (CBCT) data. Creation of a 3D maxillofacial-dental model using sequential point-matching (A) and regional surface-based registration (B). Comparison of an original 3D CBCT image of the skull model (C), initial incorporation of dental arch information into the 3D CBCT skull structure (D), and finally superimposition of the dental-skeletal data $(\mathrm{E})$.

A

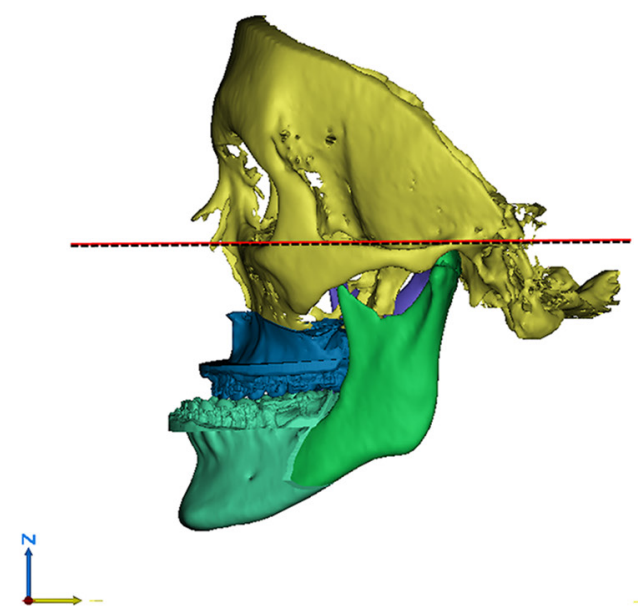

B

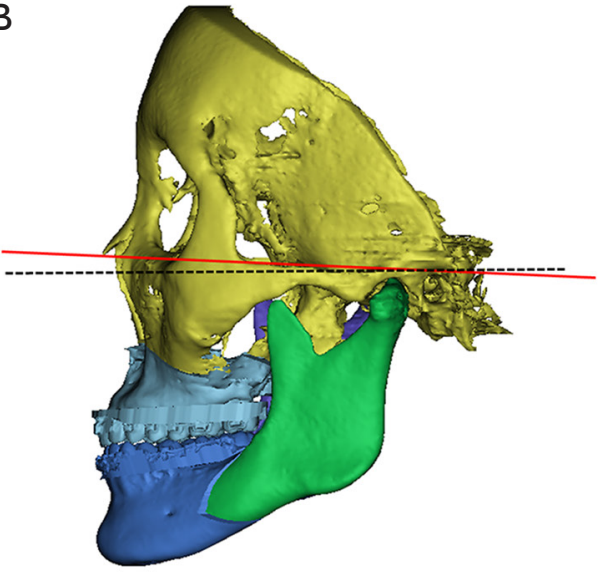

Figure 4 Setting the head position is important in VSP. It is necessary to confirm the head position of the patient before starting segment mobilization. The reference plane of the planned movement of the segment can be different for each patient. 

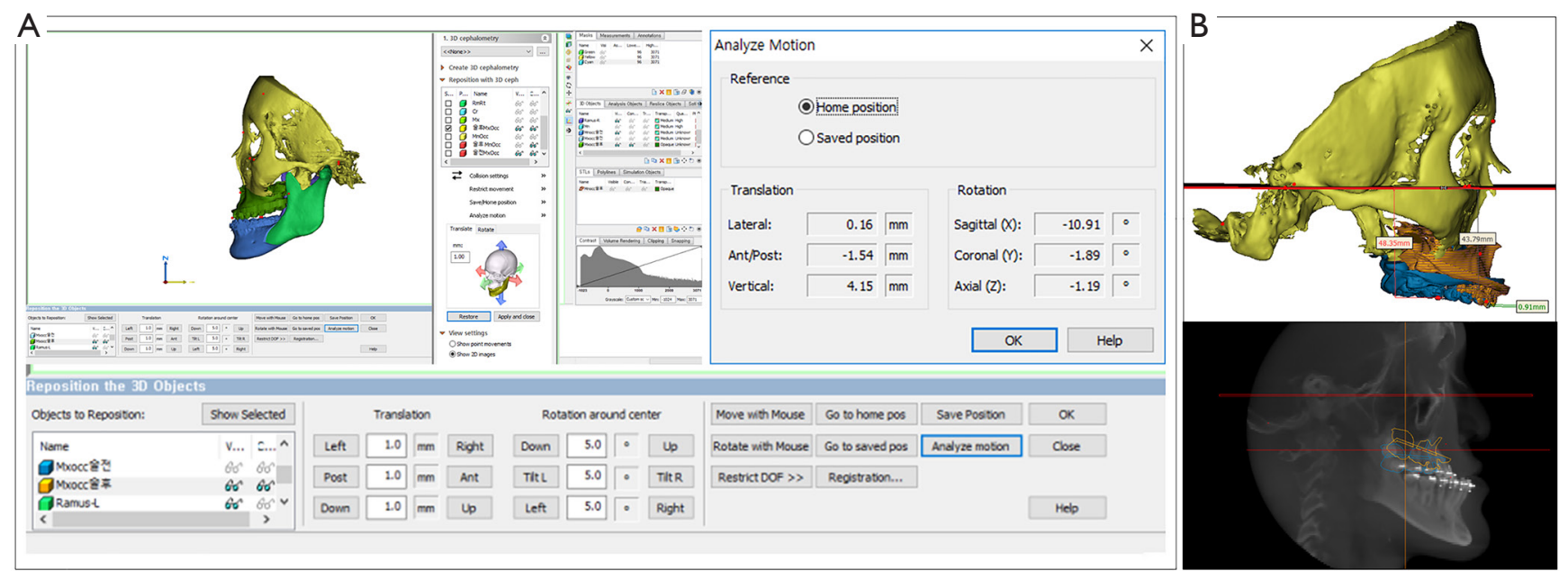

Figure 5 Segment mobilization in 3D virtual space. The anticipated position of the segments can be mobilized in virtual space (A) and the moved position is visualized with $3 \mathrm{D}$ or MPR images (B).

limitations because of reproducibility (41) or changes after mandibular set-back (42) or advancement surgery (43).

After orienting the head position in $3 \mathrm{D}$ virtual space, segmentation of the individual osseous segments is carried out by cropping the target structure and cleaning up the artifacts or unnecessary 3D structures and virtual osteotomy (11). During the VSP, the midline deviation, maxillary roll, pitch, yaw correction, and anteroposterior/vertical transverse correction need to be thoroughly confirmed. It is common to use commercially available software; such as, SimPlant $\mathrm{O} \& \mathrm{O}^{\circledR}$ (Materialise), Mimics ${ }^{\circledR}$ (Materialise N.V., Leuven, Belgium), Dolphin Imaging ${ }^{\circledR}$ (Dolphin Imaging and Management Solutions, Chatsworth, CA, USA), ProplanCMF ${ }^{\circledast}$ (Materialise), and various other software programs developed by individual institutions or local companies. According Xia et al. (2015), it is recommended to perform VSP following a specific algorithm; i.e., midline correction followed by roll and yaw correction, adjustment of the vertical position, pitch orientation, and horizontal position consecutively (11). Other institutions have suggested using an algorithm that starts with transverse correction of the midline and roll correction at first in a frontal view, then performance of pitch correction in a lateral image, and performance of maxillary yaw correction as the final stage (44). The algorithm can be modified according to the practitioner's preference and function of the module in the $3 \mathrm{D}$ simulation software.

One of the strong advantages of 3D VSP is precise visualization of the anticipating position of the segments
(Figure 5). It is easy to identify interosseous interference during the VSP process. Severe interference between the proximal and distal segments of the mandibular ramus after a planned surgery can be controlled by yaw correction of the maxillary osteotomy (44). For example, in a patient with severe facial asymmetry, canting correction can be planned with the center of rotation at the upper incisor midline. As a result, a significant gap on the deviated side would be expected. If the yaw correction of the maxilla is added in this position, the interosseous gap can be reduced (Figure 6). On the contrary to this advantage, unwanted yaw correction can be accompanied by any amount of roll correction and the midline position must be correct during canting correction of the maxilla via VSP. Therefore, after all the planned movement is finished, the position of the osteotomized segment needs to be confirmed again for every $x, y, z$ reference point (Figure 7). VSP can be used to predict and manage the interosseous interference or gap and minimize surgical morbidity and operation times (45). Severe bony interference can be corrected with the 3D VSP procedure. However, accidental mobilization in virtual space can lead to surgical error.

VSP can overcome the potential difficulties in articulator model surgery $(17,46)$ or inaccuracy of the articulator-based conventional model surgery (47). For example, when a maxillary down fracture or asymmetric maxillary impaction with down fracture on the contralateral side are needed, they are definitely accompanied by premature contact with bilateral or unilateral molars. Therefore, intermediate splint 

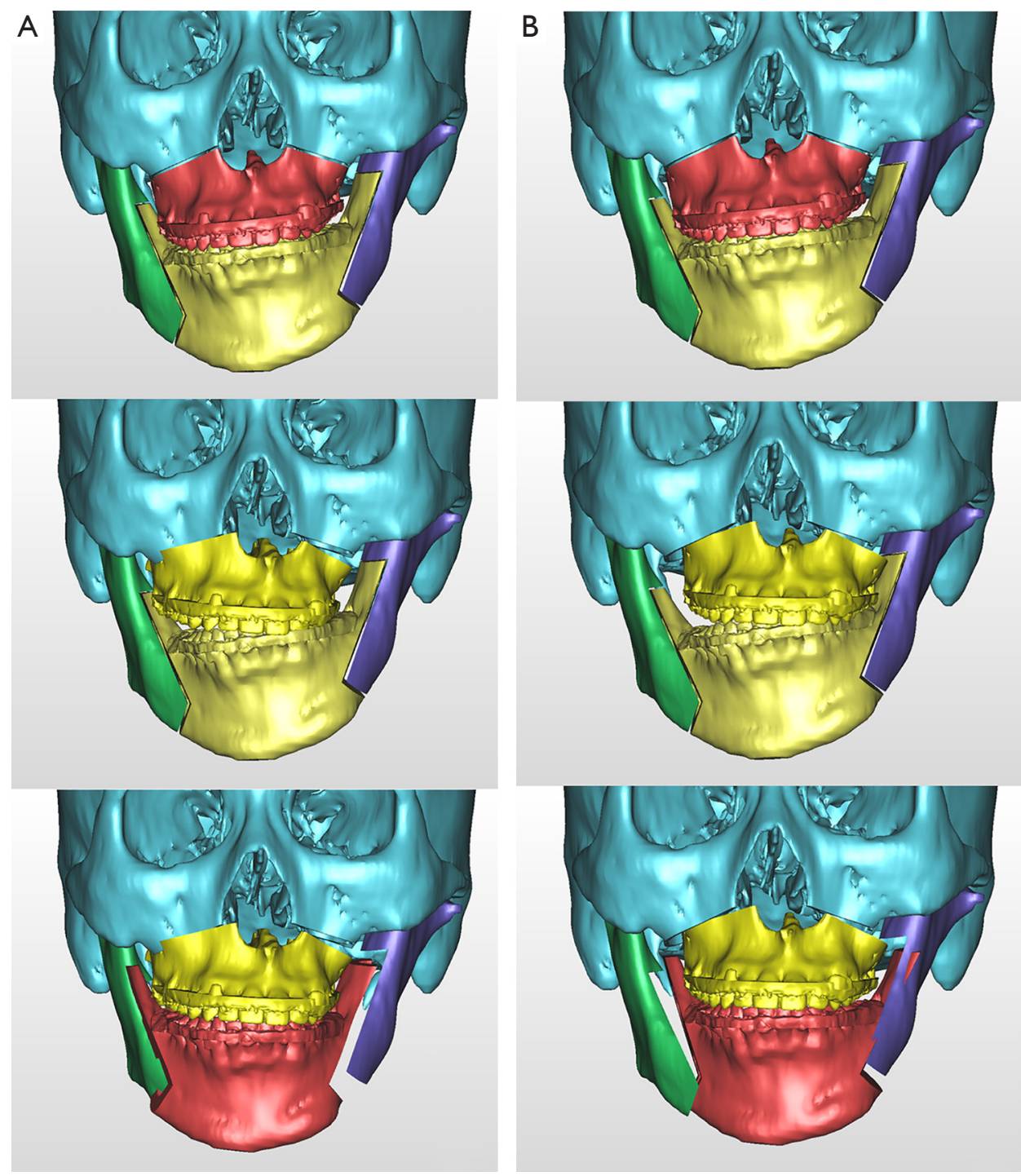

Figure 6 One example of performing a yaw correction. After maxillary canting correction with the center of rotation the upper incisor midline, there would be a significant gap on the deviated side (A). If the yaw correction of the maxilla is added, the interosseous gap can be reduced $(\mathrm{B})$.

fabrication using minimal changes in the temporomandibular joint position would be difficult with articulator-based model surgery. However, mandibular autorotation is possible in virtual space by centering the line connecting the center of the bilateral condyle head (Figure 8).

Precision in orthognathic surgery with a surgical guide and prefabricated plates

There are many reports on the accuracy and feasibility of $3 \mathrm{D}$ CAD/CAM splints. It is currently an important component of the orthognathic surgery process $(6,48-51)$.
To accurately transfer VSP to the operating room, surgical guides can also be used with or without a surgical splint (52). Surgical guides can aid two functions in surgeries; i.e., osteotomy and repositioning (53). Currently, surgical guides that utilize custom titanium miniplates showed favorable surgical accuracy (54-58). Short operating times have great advantages, but a limitation is the high cost of these systems $(54,56)$. Previously reported results have suggested that CAD/CAM surgical guides and utilization of customized titanium plates are a major trend in orthognathic surgery and can eliminate the need for 
A
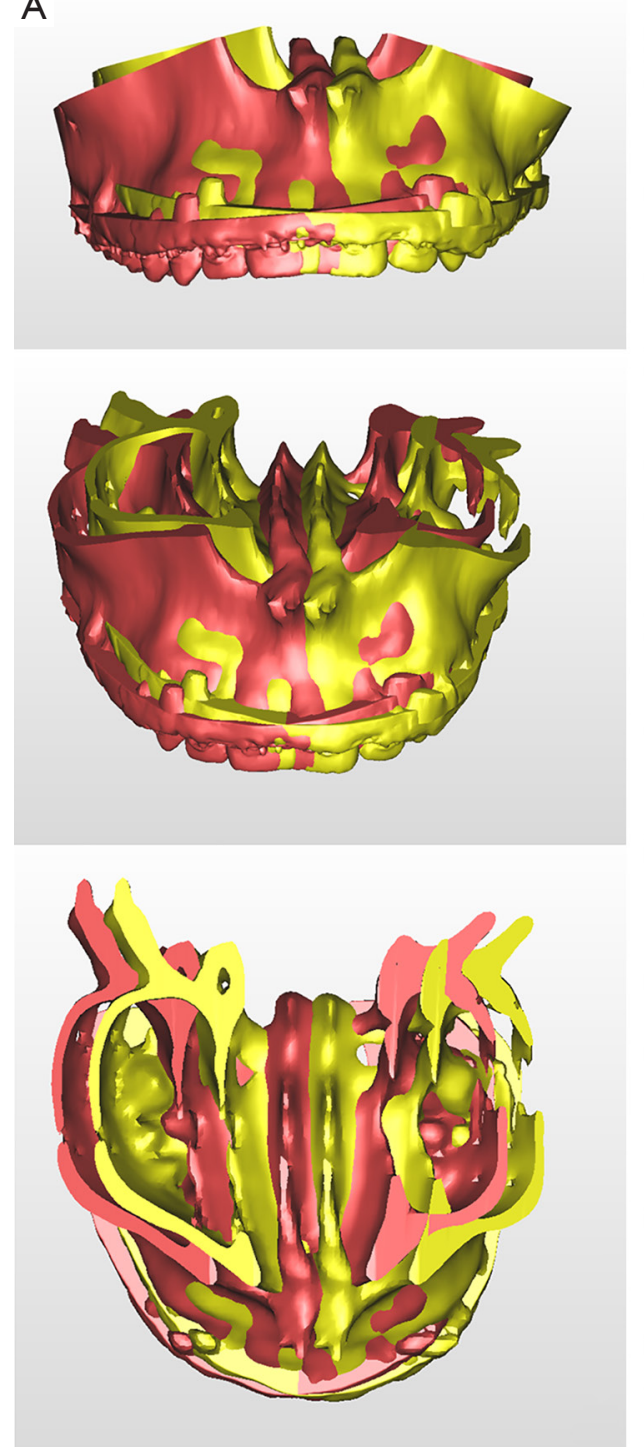

B
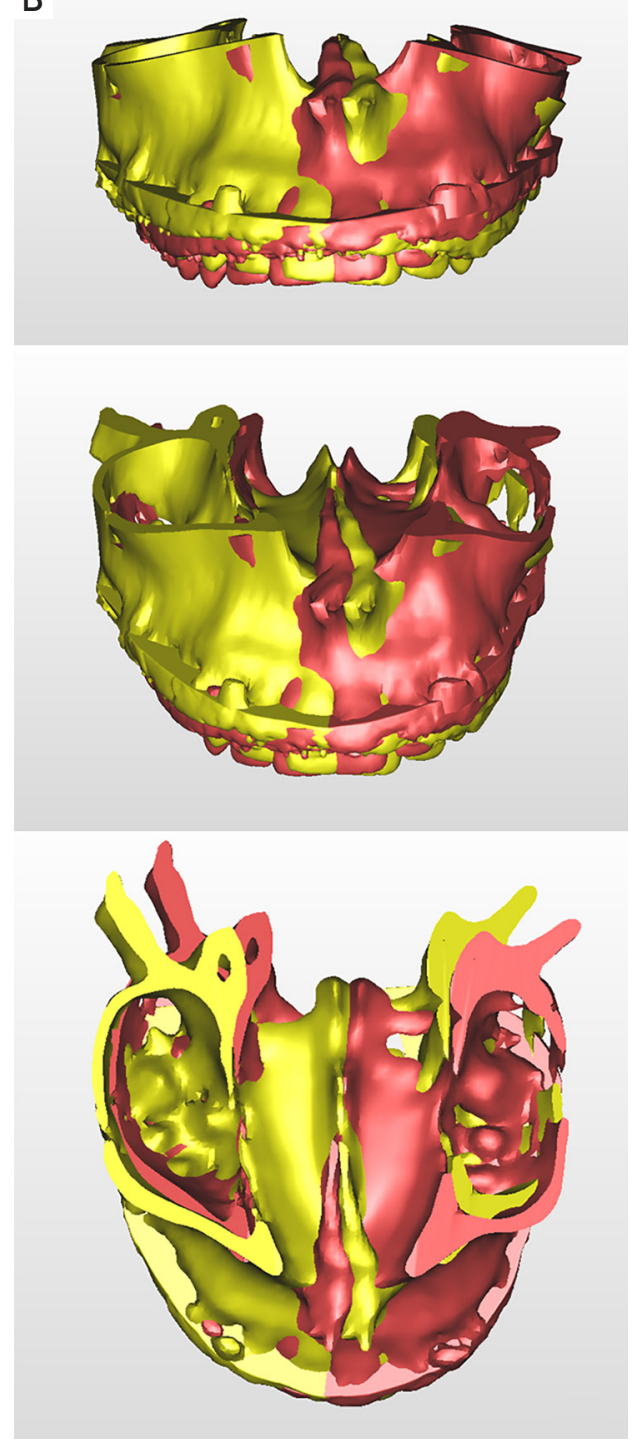

Figure 7 In maxillary canting correction, right side impaction and left side down fracture is nearly the same and there is no difference in the maxillary incisor midline (A,B). However, posterior yaw correction can be accompanied erroneously (B).

intermediate surgical pauses to reposition the maxilla.

\section{Digital workflow of VSP for orthognathic surgery using in-house processing vs. outsourcing}

The 3D software programs for VSP are usually installed at the hospital and the surgical team can design the surgical plan at each institution. Surgical plans can be accessed throughout the individual hospital via computer networks or web-based connections. Residents, patients, and operators have easy access to the $3 \mathrm{D}$ plans. This can facilitate in- depth discussion on a surgical case and activate multiple opinions on difficult cases, thereby minimizing potential mistakes. A hospital or institution that houses a laboratory that is specialized for 3D planning and CAD/CAM splint or guide fabrication is optimal for surgeons. However, considering the various obstacles in hiring experts and the flexibility in the number of the operations and management costs for 3D laboratories, outsourcing of VSP is adopted in many institutions.

$\mathrm{CAD} / \mathrm{CAM}$-assisted surgical planning in orthognathic surgery is frequently carried out by outsourcing in 

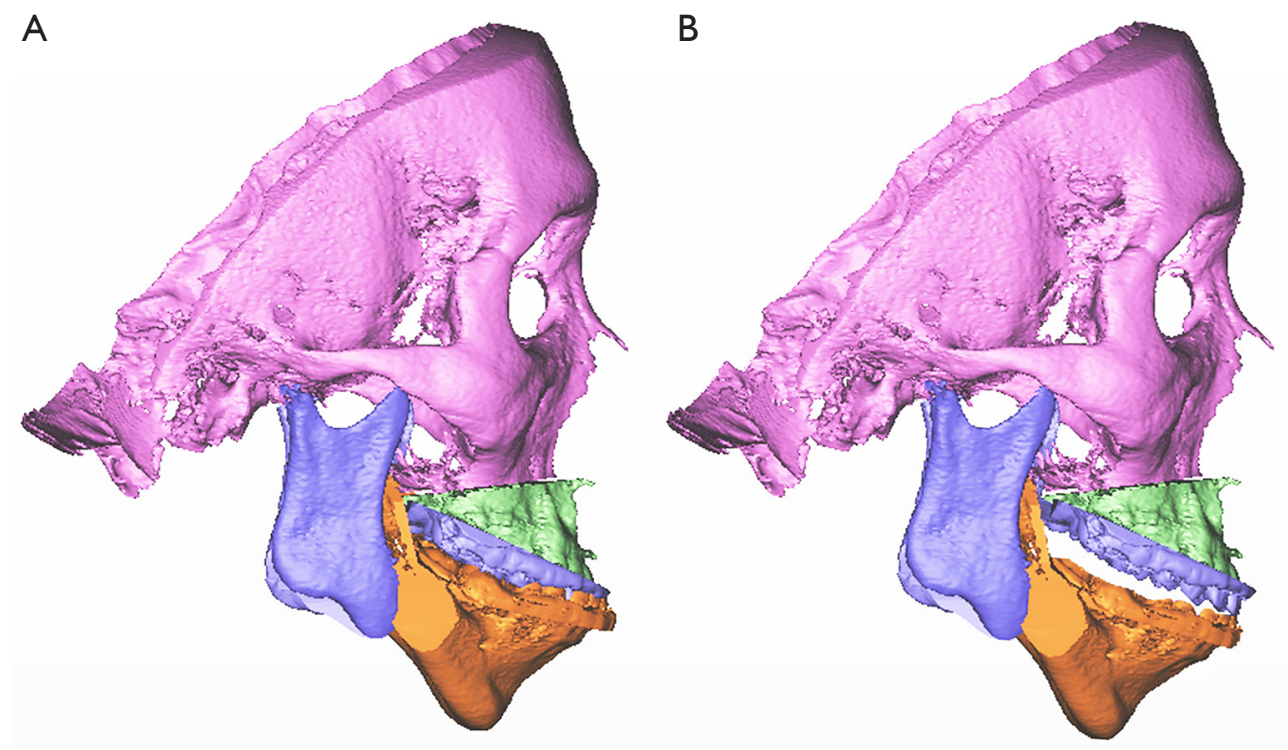

Figure 8 Virtual mandibular rotation to minimize inter-segment interference. When the maxillary downward reposition is planned (A), autorotation of mandible hinged on TMJ center is needed to fabricate an intermediate splint (B).

conjunction with a third-party vendor. Many private companies offer outsourced VSP, such as 3D Systems (VSP ${ }^{\circledR}$ Orthognathics) (59), Materialise (ProPlan CMF) (60), KLS Martin (IPS CaseDesigner ${ }^{\circledR}$ ) (13) or other domestic companies in each country or local district. In the process of establishing a surgical plan, surgeons confer with $3 \mathrm{D}$ technicians from the above mentioned companies. During an example VSP on-line meeting, a patient's intermaxillary occlusion was virtually aligned and the surgeon confirmed the finally expected occlusion after the surgery. The osteotomized segments were repositioned according to the occlusion and surgical plan. Therefore, a web-based meeting or interactive discussion is important to minimize the knowledge gap between the surgeon and technician.

The use of outsourced VSP systems is advantageous in many clinics. However, there are limitations in outsourcing, such as the high cost of processing, prolonged delivery time before surgery, and necessity for multiple web-meetings or conference calls for manufacturing outsourced CAD/ CAM-splints or surgical guides. Therefore, the need for processing VSP using in-house workflow has been suggested (61-63). 3D printed models can be fabricated from commercially available 3D printers at the clinician's institution and can reduce the preparation phase and minimize delivery time and can be used to train residents to understand $3 \mathrm{D}$ processing and simulation. It is still a demanding situation for a surgical department to hire professionals who can work faster with reasonable costs compared to an outsourced company.

There are limitations and quality of research reviewed. There can be inherent publication bias. Because of recent strong enthusiasm for application of VSP in orthognathic surgery, most of the problems are not seriously screened. Moreover, the scientific evidence are relatively low in previous literatures and there is not so many well-designed randomized control data. Studies using more objective inclusion/exclusion criteria with large number of data need to be investigated further in future research.

\section{Summary}

VSP plays an important role in orthognathic surgery in the era of digital technology. It has been suggested that VSP would be an opportunity rather than a risk factor for surgery because there is significant evidence of decreased preparation times, operation times, increased accuracy, and enhanced satisfaction of practitioners. Updates in the latest advances in computerized planning is important for surgeons who treat patients with dentofacial deformities. Understanding the benefit $v s$. limitations and cost and time efficiency $v s$. inefficiency can enhance the understanding of trends in VSP and can determine the best possible clinical settings in individual institutions.

Currently, many institutions perform VSP using 
outsourced companies. This has inherent risk of exposure of patient information and intellectual resources. This information also has a significant relationship with the development of better VSP. Moreover, all the quality assurance in VSP steps depend on the third-party and there is a gray zone to determine the exact responsibility for the surgical outcomes. To overcome the problems of outsourced VSP, in-house planning is considered to minimize communication gaps and reduce the cost and time for VSP planning. However, the decision can be made depending on the medical environment of individual institutions.

Since there are many factors determining the precision of $3 \mathrm{D}$ planning and simulation, the surgeons need to be aware that the VSP cannot automatically ensure successful surgical outcomes. Errors can be encountered at every step. Integration of $3 \mathrm{D}$ dentition to skull data, segment identification and mobilization, computer-aided surgical simulation and fabrication of splint and surgical guides, 3D image superimposition, and determination of occlusion in virtual space are factors that need consideration. Thorough understanding of the source of errors in VSP can lead to successful surgical outcomes in orthognathic surgery performed with VSP regardless of in-house or outsourced virtual planning procedures.

\section{Acknowledgments}

Funding: None.

\section{Footnote}

Reporting Checklist: The authors have completed the Narrative Review reporting checklist. Available at https://fomm. amegroups.com/article/view/10.21037/fomm-20-54/rc

Conflicts of Interest: All authors have completed the ICMJE uniform disclosure form (available at https://fomm. amegroups.com/article/view/10.21037/fomm-20-54/coif). TGK serves as an unpaid editorial board member of Frontiers of Oral and Maxillofacial Medicine from Apr 2020 to Mar 2022. The other authors have no conflicts of interest to declare.

Ethical Statement: The authors are accountable for all aspects of the work in ensuring that questions related to the accuracy or integrity of any part of the work are appropriately investigated and resolved.

Open Access Statement: This is an Open Access article distributed in accordance with the Creative Commons Attribution-NonCommercial-NoDerivs 4.0 International License (CC BY-NC-ND 4.0), which permits the noncommercial replication and distribution of the article with the strict proviso that no changes or edits are made and the original work is properly cited (including links to both the formal publication through the relevant DOI and the license). See: https://creativecommons.org/licenses/by-nc-nd/4.0/.

\section{References}

1. Swennen GR, Mollemans W, Schutyser F. Threedimensional treatment planning of orthognathic surgery in the era of virtual imaging. J Oral Maxillofac Surg 2009;67:2080-92.

2. Xia JJ, Shevchenko L, Gateno J, et al. Outcome study of computer-aided surgical simulation in the treatment of patients with craniomaxillofacial deformities. J Oral Maxillofac Surg 2011;69:2014-24.

3. Borba AM, Haupt D, de Almeida Romualdo LT, et al. How Many Oral and Maxillofacial Surgeons Does It Take to Perform Virtual Orthognathic Surgical Planning? J Oral Maxillofac Surg 2016;74:1807-26.

4. Xia J, Ip HH, Samman N, et al. Three-dimensional virtual-reality surgical planning and soft-tissue prediction for orthognathic surgery. IEEE Trans Inf Technol Biomed 2001;5:97-107.

5. Xia J, Ip HH, Samman N, et al. Computer-assisted threedimensional surgical planning and simulation: $3 \mathrm{D}$ virtual osteotomy. Int J Oral Maxillofac Surg 2000;29:11-7.

6. Haas OL Jr, Becker OE, de Oliveira RB. Computer-aided planning in orthognathic surgery-systematic review. Int J Oral Maxillofac Surg 2014;44:329-42.

7. Stokbro K, Aagaard E, Torkov P, et al. Virtual planning in orthognathic surgery. Int J Oral Maxillofac Surg 2014;43:957-65.

8. Schendel SA. Computer simulation in the daily practice of orthognathic surgery. Int J Oral Maxillofac Surg 2015;44:1451-6.

9. Shaheen E, Shujaat S, Saeed T, et al. Three-dimensional planning accuracy and follow-up protocol in orthognathic surgery: a validation study. Int J Oral Maxillofac Surg 2019;48:71-6.

10. Zinser MJ, Mischkowski RA, Sailer HF, et al. Computerassisted orthognathic surgery: feasibility study using multiple CAD/CAM surgical splints. Oral Surg Oral Med Oral Pathol Oral Radiol 2012;113:673-87.

11. Xia JJ, Gateno J, Teichgraeber JF, et al. Algorithm for 
planning a double-jaw orthognathic surgery using a computer-aided surgical simulation (CASS) protocol. Part 1: planning sequence. Int J Oral Maxillofac Surg 2015;44:1431-40.

12. Xue C, Xu H, Tian Y, et al. Precise control of maxillary multidirectional movement in Le Fort I osteotomy using a surgical guiding device. Br J Oral Maxillofac Surg 2018;56:797-804.

13. Rückschloß T, Ristow O, Muller M, et al. Accuracy of patient-specific implants and additive-manufactured surgical splints in orthognathic surgery - A threedimensional retrospective study. J Craniomaxillofac Surg 2019;47:847-53.

14. Kraeima J, Schepers RH, Spijkervet FKL, et al. Splintless surgery using patient-specific osteosynthesis in Le Fort I osteotomies: a randomized controlled multicentre trial. Int J Oral Maxillofac Surg 2020;49:454-60.

15. Zhang N, Liu S, Hu Z, et al. Accuracy of virtual surgical planning in two-jaw orthognathic surgery: comparison of planned and actual results. Oral Surg Oral Med Oral Pathol Oral Radiol 2016;122:143-51.

16. Cousley RRJ, Bainbridge M, Rossouw PE. The accuracy of maxillary positioning using digital model planning and $3 \mathrm{D}$ printed wafers in bimaxillary orthognathic surgery. $\mathrm{J}$ Orthod 2017;44:256-67.

17. Kwon TG, Choi JW, Kyung HM, et al. Accuracy of maxillary repositioning in two-jaw surgery with conventional articulator model surgery versus virtual model surgery. Int J Oral Maxillofac Surg 2014;43:732-8.

18. Ritto FG, Schmitt ARM, Pimentel T, et al. Comparison of the accuracy of maxillary position between conventional model surgery and virtual surgical planning. Int J Oral Maxillofac Surg 2018;47:160-6.

19. McCormick SU, Drew SJ. Virtual model surgery for efficient planning and surgical performance. J Oral Maxillofac Surg 2011;69:638-44.

20. Posnick JC, Adachie A, Choi E. Segmental Maxillary Osteotomies in Conjunction With Bimaxillary Orthognathic Surgery: Indications - Safety - Outcome. J Oral Maxillofac Surg 2016;74:1422-40.

21. Ismail M, Wessel J, Farrell B. Maintenance of Segmental Maxillary Expansion: The Use of Custom, Virtually Designed, and Manufactured Palatal Appliances Without the Use of an Occlusal Splint. J Oral Maxillofac Surg 2019;77:1468.e1-1468.e8.

22. Kwon TG, Miloro M, Han MD. How Accurate Is 3-Dimensional Computer-Assisted Planning for Segmental Maxillary Surgery? J Oral Maxillofac Surg
2020;78:1597-608.

23. Stokbro K, Aagaard E, Torkov P, et al. Surgical Splint Design Influences Trans-verse Expansion in Segmental Maxillary Osteotomies. J Oral Maxillofac Surg 2017;75:1249-56.

24. Tankersley AC, Nimmich MC, Battan A, et al. Comparison of the Planned Ver-sus Actual Jaw Movement Using Splint-Based Virtual Surgical Planning: How Close Are We at Achieving the Planned Outcomes? J Oral Maxillofac Surg 2019;77:1675-80.

25. Cevidanes LH, Tucker S, Styner M, et al. Threedimensional surgical simulation. Am J Orthod Dentofacial Orthop 2010;138:361-71.

26. Hsu PJ, Denadai R, Pai BCJ, et al. Outcome of facial contour asymmetry after conventional two-dimensional versus computer-assisted three-dimensional planning in cleft orthognathic surgery. Sci Rep 2020;10:2346.

27. Plooij JM, Maal TJ, Haers P, et al. Digital threedimensional image fusion processes for planning and evaluating orthodontics and orthognathic surgery. A systematic review. Int J Oral Maxillofac Surg 2011;40:341-52.

28. Swennen GR, Barth EL, Eulzer C, et al. The use of a new 3D splint and double CT scan procedure to obtain an accurate anatomic virtual augmented model of the skull. Int J Oral Maxillofac Surg 2007;36:146-52.

29. Swennen GR, Mollemans W, De Clercq C, et al. A conebeam computed tomography triple scan procedure to obtain a three-dimensional augmented virtual skull model appropriate for orthognathic surgery planning. J Craniofac Surg 2009;20:297-307.

30. Gateno J, Xia J, Teichgraeber JF, et al. A new technique for the creation of a computerized composite skull model. J Oral Maxillofac Surg 2003;61:222-7.

31. Uechi J, Tsuji Y, Konno M, et al. Generation of virtual models for planning orthognathic surgery using a modified multimodal image fusion technique. Int J Oral Maxillofac Surg 2015;44:462-9.

32. Bobek S, Farrell B, Choi C, et al. Virtual surgical planning for orthognathic surgery using digital data transfer and an intraoral fiducial marker: the charlotte meth-od. J Oral Maxillofac Surg 2015;73:1143-58.

33. Sun Y, Luebbers HT, Agbaje JO, et al. Accuracy of upper jaw positioning with intermediate splint fabrication after virtual planning in bimaxillary orthognathic surgery. J Craniofac Surg 2013;24:1871-6.

34. Lin HH, Chiang WC, Lo LJ, et al. Artifact-resistant superimposition of digital dental models and cone-beam 
computed tomography images. J Oral Maxillofac Surg 2013;71:1933-47.

35. Nkenke E, Zachow S, Benz M, et al. Fusion of computed tomography data and optical 3D images of the dentition for streak artefact correction in the simulation of orthognathic surgery. Dentomaxillofac Radiol 2004;33:226-32.

36. Kim BC, Lee CE, Park W, et al. Integration accuracy of digital dental models and 3-dimensional computerized tomography images by sequential point- and surface-based markerless registration. Oral Surg Oral Med Oral Pathol Oral Radiol Endod 2010;110:370-8.

37. Noh H, Nabha W, Cho JH, et al. Registration accuracy in the integration of laser-scanned dental images into maxillofacial cone-beam computed tomography images. Am J Orthod Dentofacial Orthop 2011;140:585-91.

38. de Waard O, Baan F, Verhamme L, et al. A novel method for fusion of intra-oral scans and cone-beam computed tomography scans for orthognathic surgery planning. J Craniomaxillofac Surg 2016;44:160-6.

39. Park JH, Hwang CJ, Choi YJ, et al. Registration of digital dental models and cone-beam computed tomography images using 3-dimensional planning software:

Comparison of the accuracy according to scanning methods and software. Am J Orthod Dentofacial Orthop 2020;157:843-51.

40. Zhu S, Keeling A, Hsung TC, et al. The difference between registered natural head position and estimated natural head position in three dimensions. Int J Oral Maxillofac Surg 2018;47:276-82.

41. Yang HJ, Han JJ, Hwang SJ. Accuracy of 3D reproduction of natural head position using three different manual reorientation methods compared to $3 \mathrm{D}$ software. J Craniomaxillofac Surg 2018;46:1625-30.

42. Cho D, Choi DS, Jang I, et al. Changes in natural head position after orthognathic surgery in skeletal Class III patients. Am J Orthod Dentofacial Orthop 2015;147:747-54.

43. Lin $X$, Edwards SP. Changes in natural head position in response to mandibular advancement. Br J Oral Maxillofac Surg 2017;55:471-5.

44. Lonic D, Pai BC, Yamaguchi K, et al. Computer-Assisted Orthognathic Surgery for Patients with Cleft Lip/Palate: From Traditional Planning to Three-Dimensional Surgical Simulation. PLoS One 2016;11:e0152014.

45. Valls-Ontañón A, Ascencio-Padilla RDJ, Vela-Lasagabaster A, et al. Relevance of 3D virtual planning in predicting bony interferences between distal and proximal fragments after sagittal split osteotomy. Int J Oral Maxillofac Surg 2020;49:1020-8.

46. Hsu SS, Gateno J, Bell RB, et al. Accuracy of a computeraided surgical simulation protocol for orthognathic surgery: a prospective multicenter study. J Oral Maxillofac Surg 2013;71:128-42.

47. Lartizien R, Zaccaria I, Noyelles L, et al. Quantification of the inaccuracy of conventional articulator model surgery in Le Fort 1 osteotomy: evaluation of 30 patients controlled by the Orthopilot((R)) navigation system. Br J Oral Maxillofac Surg 2019;57:672-7.

48. Metzger MC, Hohlweg-Majert B, Schwarz U, et al. Manufacturing splints for orthognathic surgery using a three-dimensional printer. Oral Surg Oral Med Oral Pathol Oral Radiol Endod 2008;105:e1-7.

49. Choi JY, Song KG, Baek SH. Virtual model surgery and wafer fabrication for orthognathic surgery. Int J Oral Maxillofac Surg 2009;38:1306-10.

50. Bai S, Bo B, Bi Y, et al. CAD/CAM surface templates as an alternative to the intermediate wafer in orthognathic surgery. Oral Surg Oral Med Oral Pathol Oral Radiol Endod 2010;110:e1-7.

51. Chin SJ, Wilde F, Neuhaus M, et al. Accuracy of virtual surgical planning of or-thognathic surgery with aid of CAD/ CAM fabricated surgical splint-A novel 3D ana-lyzing algorithm. J Craniomaxillofac Surg 2017;45:1962-70.

52. Zinser MJ, Sailer HF, Ritter L, et al. A paradigm shift in orthognathic surgery? A comparison of navigation, computer-aided designed/computer-aided manufactured splints, and "classic" intermaxillary splints to surgical transfer of virtual orthognathic planning. J Oral Maxillofac Surg 2013;71:2151.e1-21.

53. Li B, Zhang L, Sun H, et al. A novel method of computer aided orthognathic surgery using individual CAD/CAM templates: a combination of osteotomy and repositioning guides. Br J Oral Maxillofac Surg 2013;51:e239-44.

54. Heufelder M, Wilde F, Pietzka S, et al. Clinical accuracy of wafer less maxillary positioning using customized surgical guides and patient specific osteosynthesis in bimaxillary orthognathic surgery. J Craniomaxillofac Surg 2017;45:1578-85.

55. Brunso J, Franco M, Constantinescu T, et al. CustomMachined Miniplates and Bone-Supported Guides for Orthognathic Surgery: A New Surgical Procedure. J Oral Maxillofac Surg 2016;74:1061.e1-1061.e12.

56. Hanafy M, Akoush Y, Abou-ElFetouh A, et al. Precision of orthognathic digital plan transfer using patient-specific cutting guides and osteosynthesis versus mixed analogue- 
digitally planned surgery: a randomized controlled clinical trial. Int J Oral Maxillofac Surg 2020;49:62-8.

57. Mazzoni S, Bianchi A, Schiariti G, et al. Computeraided design and computer-aided manufacturing cutting guides and customized titanium plates are useful in upper maxilla waferless repositioning. J Oral Maxillofac Surg 2015;73:701-7.

58. Kraeima J, Jansma J, Schepers RH. Splintless surgery: does patient-specific CAD-CAM osteosynthesis improve accuracy of Le Fort I osteotomy? Br J Oral Maxillofac Surg 2016;54:1085-9.

59. Gray R, Gougoutas A, Nguyen V, et al. Use of threedimensional, CAD/CAM-assisted, virtual surgical simulation and planning in the pediatric craniofacial population. Int J Pediatr Otorhinolaryngol 2017;97:163-9.

doi: $10.21037 /$ fomm-20-54

Cite this article as: Kwon TG, Lee ST, Choi SY, Kim JW. Considerations for virtual surgical planning and simulation in orthognathic surgery-a narrative review. Front Oral Maxillofac Med 2020;2:27.
60. Knoops PGM, Borghi A, Breakey RWF, et al. Threedimensional soft tissue pre-diction in orthognathic surgery: a clinical comparison of Dolphin, ProPlan CMF, and probabilistic finite element modelling. Int J Oral Maxillofac Surg 2019;48:511-8.

61. Hatamleh MM, Ong J, Hatamleh ZM, et al. Developing an In-house Interdisciplinary Three-Dimensional Service: Challenges, Benefits, and Innovative Health Care Solutions. J Craniofac Surg 2018;29:1870-5.

62. Marschall JS, Dutra V, Flint RL, et al. . In-House Digital Workflow for the Management of Acute Mandible Fractures. J Oral Maxillofac Surg 2019;77:2084.e1-2084.e9.

63. Abo Sharkh H, Makhoul N. In-House Surgeon-Led Virtual Surgical Planning for Maxillofacial Reconstruction. J Oral Maxillofac Surg 2020;78:651-60. 